\title{
3D Printing of Functional Biomaterials for Tissue Engineering
}

\author{
Wei Zhu ${ }^{1}$, Xuanyi $\mathrm{Ma}^{2}$, Maling $\mathrm{Gou}^{3}$, Deqing $\mathrm{Mei}^{4}$, Kang Zhang ${ }^{5}$, Shaochen Chen $*^{1,2}$ \\ ${ }^{1}$ NanoEngineering Department, ${ }^{2}$ Bioengineering Department, University of California, \\ San Diego, USA \\ ${ }^{3}$ State Key Laboratory of Biotherapy and Cancer Center, West China Hospital, Sichuan \\ University, and Collaborative Innovation Center for Biotherapy, Chengdu, China \\ ${ }^{4}$ Department of Mechanical Engineering, Zhejiang University, China \\ ${ }^{5}$ Shiley Eye Center and Institute for Genomic Medicine, University of California, San \\ Diego, USA \\ * Corresponding author: Chen, Shaochen (chen168@eng.ucsd.edu)
}

\begin{abstract}
$3 \mathrm{D}$ printing is emerging as a powerful tool for tissue engineering by enabling $3 \mathrm{D}$ cell culture within complex 3D biomimetic architectures. This review discusses the prevailing 3D printing techniques and their most recent applications in building tissue constructs. The work associated with relatively well-known inkjet and extrusion-based bioprinting is presented with the latest advances in the fields. Emphasis is put on introducing two relatively new light-assisted bioprinting techniques, including digital light processing (DLP)-based bioprinting and laser based two photon polymerization (TPP) bioprinting. 3D bioprinting of vasculature network is particularly discussed for its foremost significance in maintaining tissue viability and promoting functional maturation. Limitations to current bioprinting approaches, as well as future directions of bioprinting functional tissues are also discussed.
\end{abstract}

\section{Introduction}

Three-dimensional (3D) printing has led to significant advancements in many areas in the past two decades, including aerospace, consumer products, arts, food industry and manufacturing [1]. With the recent advances of 3D printing technologies, a growing number of researchers in the biomedical engineering field are employing 3D printing as a transformative tool for biomedical applications, especially for tissue engineering and regenerative medicine. Tissue engineering is an emerging field that aims to develop biological substitutes of native human tissues or organs for in vitro drug screening to decrease the use of animals and increase the reliability of testing results, or for in vivo transplantation to mitigate the organ shortage and transplantation need. Recent research has greatly increased awareness of the dramatic differences in cell behavior between 2D and 3D culture systems. Culturing cells in 3D provides a more physiologically relevant environment to guide cell behaviors and enhance their functions 
[2-5]. Therefore, great efforts have been made to develop 3D biofabrication techniques that can generate complex, functional 3D architectures with appropriate biomaterials and cell types to mimic the native micro-environment and biological components.

In this review, we discuss the most dominant and important examples of 3D printing modalities and their applications in tissue engineering and relevant biomedical research. While cell source and biomaterial are two other key components in 3D biofabrication for tissue engineering, we will focus on the 3D biofabrication platforms and their end products to present the state-of-the-art 3D bioprinting capability. More discussion on the choice of cell types and biomaterials can be found in a recent review by Murphy et al. [1]. We first introduce the current prevailing 3D bioprinting techniques, including inkjet bioprinting, extrusion bioprinting and light-assisted bioprinting. Examples of the printed scaffolds are presented to demonstrate the printing capability as well as the limitations. Emphasis is put on the recently developed light-assisted bioprinting systems, including digital light processing (DLP)-based bioprinting and laser-based bioprinting, for their superior printing speed and resolution. Then we specifically discuss 3D printing of vascularized tissues, as this represents one of the most fundamental challenges in tissue engineering. Lastly, we summarize the challenges and future directions of $3 \mathrm{D}$ bioprinting for tissue engineering.

\section{Overview}

A majority of the traditional methods for creating 3D scaffolds-- including electrospinning [6], freeze-drying [7], gas foaming [8,9], particle or porogen leaching [10,11]-- have control only on the bulk properties of the scaffolds and do not allow precise control of the internal architecture and topology [12]. Inspired by photolithography in electronics manufacturing, photomasks were used to dictate the polymerization of photosensitive biomaterials to create 3D cell-laden hydrogel scaffolds $[13,14]$. In this method, multiple photomasks are needed for the alteration of designs or parameters, which is costly and time consuming. A mold made of polydimethylsiloxane (PDMS) was also employed to confine cell-laden gel into the dictated 3D structure [15]. Similarly, this method requires different molds for varying the design of the 3D structures. 3D printing, assisted with computer aided design (CAD) technology, requires no physical masks or molds for creating the internal architecture, thus serving as a transformative tool for creating complex 3D structures at micro- and even nanoscale with lower cost and higher flexibility and efficiency. There are three major technologies used for 3D printing biomaterials and cells: inkjet bioprinting, extrusion bioprinting and light-assisted bioprinting.

\section{Inkjet and extrusion based bioprinting}

For the platforms to be biocompatible with cell work, printing systems and technologies that were used in non-biological applications have been modified to deal with biocompatible materials and to minimize any potential compromise on cell viability $[16,17]$. The early development of the bioprinting systems to print cellular assemblies that mimic their respective architecture in organs originated from modifying commercially available inkjet printers [16]. Instead of dispensing ink, these inkjet printers were modified to dispense protein or cell solutions [16]. Although this printing 
system is automated for the high-throughput manufacture of cell arrays, it is still limited to 2D tissue constructs. By using thermosensitive gels in the same system, one could print successive layers of cellular aggregates followed by fusion of the aggregates, to generate 3D cell-laden constructs [18]. Further advancement of inkjet printing systems over the decade allowed the biofabrication of complex and heterogeneous 3D tissue constructs consisting of multiple cell types (Figure 1a-c) [19]. The thermal inkjet printers are widely available at relatively low cost, but low droplet directionality, nozzle clogging and the risk of exposing cells to thermal and mechanical stress pose considerable concerns to the use of these printers in 3D bioprinting.

Extrusion-based bioprinting systems have also been extensively developed to produce bio-constructs with cells. Following the first use of inkjet printing systems for cell applications, a robotic solid freeform fabrication platform with a gel deposition tool, such as an extrusion-based system, was developed to deposit pre-seeded alginate hydrogel layer by layer to produce 3D pre-seeded living implants of arbitrary geometries [17]. The successful application of the technology in printing cell-laden construct further leads to future organ printing applications [20,21]. Multicellular spheroids were used as a building block for such applications, which eventually lead to a layer-by-layer robotic biofabrication of 3D functional living macro-tissues and organ constructs [20]. In addition to spheroid and organoid printing, extrusion-based bioprinting systems have also been used in recent years in combination with novel biomaterials to create liver, cartilage and neural tissue construct [22-24]. Recent demonstrations of nozzle-based bioprinting technology in patterning de-cellularized extracellular matrix (dECM) further highlight the future potential of this bioprinting technology in manipulating biomimetic materials with complex composition (Figure 1d-f) [25]. In addition, a bionic ear was created via the 3D printing of a cell-seeded hydrogel matrix in the anatomic geometry of a human, along with an intertwined conducting polymer consisting of infused silver nanoparticles (Figure $1 \mathrm{~g}$ ) [26]. Due to the excellent electro-conductivity of nanoparticles, the bionic ear exhibits enhanced auditory sensing for radio frequency signals as well as stereo audio music.

Inkjet and extrusion bioprinters have unique advantages in terms of simplicity, flexibility and low cost. However, these two methods have limitations as well. First of all, cell damage and death as well as cell sedimentation and aggregation exist in both methods due to the shear stress and the small orifice diameter of the nozzles used to deliver the bio-ink. Also, the printing resolution is limited by the physical confinement of the nozzles, usually above $50 \mu \mathrm{m}$. Moreover, the structural integrity of the printed structures is another obstacle, especially at the interfaces of droplets (in the case of inkjet printing) and lines (in the case of extrusion printing).

\section{Light-assisted bioprinting}

In addition to inkjet printing and extrusion-based printing technology, light-assisted bioprinting platforms are increasingly being used for cell printing and tissue engineering applications. These systems mostly involve the use of photo-polymerization of biomaterials and can print a variety of cell types with good cell viability $[27,28]$. There 
are two sub groups of light-assisted bioprinting systems: digital light processing (DLP)based printers and laser-based printers.

As shown in Figure 2a, the DLP printer developed by Lu et al. and further improved by Zhang et al. - the dynamic optical projection stereolithography (DOPsL) platform utilizes a digital micromirror device (DMD) chip, composed of approximately one million micromirrors, to modulate the UV light and project an optical pattern-dictated by the custom-designed computer-aided design (CAD) model-onto the photopolymer solution $[29,30]$. The resolution of this printer is dictated by the focal size of the light beam from each micromirror, which is at micron scale $[27,31]$. Compared to the serial printing process (drop-by-drop or line-by-line) of the inkjet or extrusion printers, the DLP printer prints parallelly by projecting the entire plane of optical pattern onto the photopolymer solution, which significantly reduces the time required for the fabrication[30]. And by continuously refreshing the projected optical patterns and moving the stage with the printed object, smooth 3D objects can be printed (as shown in Figure $2 \mathrm{~b}$ ) with no artificial interfaces which occur between the droplets (in the inkjet printing) or the lines (in the extrusion printing)[30,32]. By eliminating the interfacial artifacts, the mechanical integrity of the printed 3D objects can be greatly improved. Due to these advantages, the DOPsL system has been employed to create a variety of complex 3D structures such as domes (Figure 2b), vasculature network (Figure 2c), and neuronal conduits (Figure 2d), with different biomaterials including polyethylene glycol diacrylate (PEGDA), glycidyl methacrylate-modified hyaluraunic acid (GM-HA), gelatin methacrylate (GelMa) as well as cells [5,27,30,33-36]. Additionally, functional nanoparticles can be incorporated into the biomaterials to fabricate functional biomedical devices via DOPsL printing[37,38]. Gou et al. presented a liver-inspired 3D detoxification device, which was generated by 3D printing of designer hydrogels with polydiacetylene (PDA) nanoparticles installed in the hydrogel matrix (Figure 2e). The functional nanoparticles could attract, capture and sense toxins, while the 3D matrix with a modified liver lobule microstructure allowed toxins to be trapped efficiently [37].

Laser-based printing has different variations, including laser direct writing (LDW) [39], laser-induced forward transfer (LIFT) [40], matrix-assisted pulsed laser evaporation (MAPLE) and so on [41], where a laser beam is focused through a high magnification objective lens to induce polymerization or material transfer on the sample slide [12]. Combined with the 3D movement of the sample stage, complex 3D structures can be fabricated at a submicron resolution. As shown in Figure 3a, LIFT was used to create a multi-layered, fully cellurized skin substitute with fibroblasts and keratinocytes. The printed skin constructs were transplanted in vivo in mice and successful integration with the host skin tissue was observed [28].

One special type of LDW system is two-photon polymerization (TPP), which utilizes a focused near-infrared femtosecond laser with a wavelength of $800 \mathrm{~nm}$ to induce a nonlinear optical effect - two-photon absorption - and lead to the polymerization of the monomer solution [42]. Because two-photon absorption only happens in the center region of the laser focal spot where the energy is above the threshold to trigger this nonlinear effect, TPP can provide a very high resolution beyond the diffraction limit. 
Figure 3b shows a typical TPP setup [42]. A line array with $400 \mathrm{~nm}$ line width and a 3D woodpile structure with $1 \mu \mathrm{m}$ line width were fabricated using PEGDA [42]. In addition, free suspending webs with special designs were fabricated to study the single cell response to negative and positive Poisson's ratios (Figure 3c) [43].

While light-assisted bioprinting possesses numerous advantages, such as good biocompatibility, high resolution and great efficiency, there still remain some challenges to be addressed. First of all, the material choice for the light-assisted printing is limited to the photosensitive polymers, which prevents the use of many biomaterials and requires additional chemical modifications to make the materials photopolymerizable. Also, with no nozzles (used in the inkjet and extrusion printers) to deliver the material to the desired region for fabrication, the photopolymers are usually filled within the entire reservoir where $3 \mathrm{D}$ objects are printed including the space not intended for polymerization, which raises the concern of wasting materials and increasing the cost. Table 1 summarizes the different bioprinting techniques we discussed above.

\section{D printing of vascularized tissues}

Creating functional vasculature represents one of the most fundamental challenges in tissue engineering. For large engineered tissue constructs, vascularization is pivotal for maintaining viability, especially in the case of highly metabolic cardiac tissue $[44,45]$. Strategies to stimulate the vascularization of implanted tissue substitutes include chemical modification of biomaterials, optimization of pore sizes to facilitate blood vessel ingrowth, and incorporation of pro-angiogenic growth factors. However, the recruitment of native endothelial cells and subsequent physiological growth of new blood vessels occurs at a prohibitively long time-scale, preventing sufficient mass transfer throughout the large constructs during the first days after implantation [46]. As an alternate approach, 3D printing vasculature in vitro has drawn great interest in the tissue engineering field, with the rationale that a preformed microvascular network can better anastomose to the host circulation to achieve functional perfusion within the implant $[44,46]$.

To create the lumen of the vasculature network, one method is to use a sacrificial ink to create the 3D interconnecting network, which can be removed after building the surrounding construct, leaving hollow channels for the perfusion of endothelial cells for endothelialization of the blood vessels. Miller et al. reported a carbohydrate glass that can be thermally extruded into a 3D lattice and rapidly dissolved in the environment biocompatible for living cells (Figure 4a\&b). Combining 3D extrusion printing and cast molding, they created 3D interconnecting perfusable vasculature and incorporated it into an engineered hepatic tissue to sustain the metabolic function of primary rat hepatocytes (Figure 4c) [47]. The temperature for printing the carbohydrate glass is above $100{ }^{\circ} \mathrm{C}$, which prevents the simultaneous printing of cells. The cast molding technique is limited to the construction of simple block tissue architectures. Kolesky et al. developed another aqueous fugitive ink composed of Pluronic F127 which can be printed and dissolved under mild conditions, which is biologically compatible to cells. This property enables the co-printing of cell-loaded inks. Using a 3D bioprinter with four 
print-heads, heterogeneous cell-laden tissue constructs with interconnecting vasculature network were printed with multiple cell types [48].

Efforts have also been made to print 3D vasculature network directly without any pre/post fabrication process. Zhang et al. recently reported the direct bioprinting of vessel-like cellular microfluidic channels with hydrogels, such as alginate and chitosan (Figure 4d) [49]. This is achieved by using a coaxial nozzle with laminar flow of the hydrogel and the crosslinker, which forms the hollow tubes in the interface. Cells (cartilage progenitor cells) are also encapsulated in the hydrogel to demonstrate the capability of direct printing cell-laden tissues with perusable vasculature network (Figure $4 \mathrm{e} \& \mathrm{f})$. Yu et al. further combined this printing method with the direct printing of polymerfree cellular strands as the demonstration of a hybrid bioprinting approach for scale-up vascularized tissue fabrication. Fibroblast tissue strands were co-printed with the vasculature network, and complete fusion and maturation of the tissues were observed in seven days [50].

\section{Discussion}

Although at its early developmental stage, 3D bioprinting is emerging as a promising tool in the tissue engineering field, providing bioengineering researchers with the unprecedented capability to engineer complex 3D biological architectures. Significant progress has been achieved in developing powerful biofabrication systems to meet various requirements of bioprinting intricate biomimetic structures with a variety of biomaterials and cells as discussed in the prior sections. However, there are still significant technological challenges for the successful development of functional tissue or organ substitutes. To better control the physical guidance provided by the microarchitecture and the heterogeneous distribution of functional biomolecules-- such as growth factors, peptide ligands and so on-- resolution needs to be improved to the subcellular or molecular level (nanometer scale) for most of the existing bioprinters. Additionally, to provide the essential physical, chemical and biological cues to the printed cells, the biocompatibility of the bioprinter to a variety of biomaterials needs to be extended, since the biomaterials act as the direct interface with the cells [51]. Combinational use of bioprinters with different working principles can be a viable solution. A majority of the current bioprinters are developed for lab research use and the printed tissues are much smaller than clinically relevant sizes. Further improvements to the printing capability and speed are in great need for large scale tissue production for clinical uses. As the size of the printed tissue increases, nutrition and oxygen transportation will become more of a concern to maintain the viability of the cells and promote the maturation of the tissue, thus requiring successful vascularization of the tissue. Although significant progress has been made to bioprint vasculature network, the maturation and integration of such vasculature network throughout the entire tissue remains a challenge. Dynamic culture using bioreactors could be a solution to promote the maturation and prolong the viability of the vascularized tissue. For in vivo transplantation, the tissue should also have the appropriate interface with sufficient mechanical properties to suture to the host circulation and withstand the pulsatile pressure of the blood flow [52]. In summary, bioprinting functional tissues requires efforts from multiple fields, including manufacturing, material science, biology and 
medicine. Interdisciplinary collaborations from these fields are needed to address the challenges.

\section{Acknowledgements}

This project is supported in part by grants from the California Institute for Regenerative Medicine (Grant no. RT3-07899), the National Institutes of Health (Grant no. EB017876, EB012597), Department of Defense (Grant no. W81XWH-14-1-0522), and the National Science Foundation (Grants no. 1547005 and 1332681).

\section{References and recommended reading}

Papers of particular interest, published within the period of review, have been highlighted as:

- of special interest

.. of outstanding interest

1. Murphy S V, Atala A: 3D bioprinting of tissues and organs. Nat. Biotechnol. 2014, 32:773-785.

- Comprehensive review on 3D bioprinting strategies, ideal material properties and cell sources for tissue and organ engineering.

2. Thoma CR, Zimmermann M, Agarkova I, Kelm JM, Krek W: 3D cell culture systems modeling tumor growth determinants in cancer target discovery. Adv. Drug Deliv. Rev. 2014, 69-70:29-41.

3. Amann A, Zwierzina M, Gamerith G, Bitsche M, Huber JM, Vogel GF, Blumer M, Koeck S, Pechriggl EJ, Kelm JM, et al.: Development of an innovative 3D cell culture system to study tumour - Stroma interactions in non-small cell lung cancer cells. PLoS One 2014, 9.

4. Puschmann TB, Zandén C, De Pablo Y, Kirchhoff F, Pekna M, Liu J, Pekny M: Bioactive 3D cell culture system minimizes cellular stress and maintains the in vivo-like morphological complexity of astroglial cells. Glia 2013, 61:432440.

5. Ma X, Qu X, Zhu W, Li Y-S, Yuan S, Zhang H, Liu J, Wang P, Lai CSE, Zanella F, et al.: Deterministically patterned biomimetic human iPSC-derived hepatic model via rapid 3D bioprinting. Proc. Natl. Acad. Sci. 2016, 113:2206-2211.

- This paper reports the application of the DLP based bioprinter to the development of an in vitro hepatic model that mimics the several in vivo features of liver. Human iPSCderived hepatic cells were bioprinted with supporting cells in a biomimetic liver lobule pattern.

6. Fong ELS, Lamhamedi-Cherradi S-E, Burdett E, Ramamoorthy V, Lazar AJ, Kasper FK, Farach-Carson MC, Vishwamitra D, Demicco EG, Menegaz B a, et al.: Modeling Ewing sarcoma tumors in vitro with 3D scaffolds. Proc. Natl. 
Acad. Sci. U. S. A. 2013, 110:6500-5.

7. Lee MK, Rich MH, Lee J, Kong H: A bio-inspired, microchanneled hydrogel with controlled spacing of cell adhesion ligands regulates 3D spatial organization of cells andtissue. Biomaterials 2015, 58:26-34.

8. Jiang J, Carlson MA, Teusink MJ, Wang H, MacEwan MR, Xie J: Expanding Two-Dimensional Electrospun Nanofiber Membranes in the Third Dimension By a Modified Gas-Foaming Technique. ACS Biomater. Sci. Eng. 2015, 1:9911001.

9. Bajaj P, Schweller RM, Khademhosseini A, West JL, Bashir R: 3D Biofabrication Strategies for Tissue Engineering and Regenerative Medicine. Annu. Rev. Biomed. Eng. 2014, 16:247-76.

10. Zhang J, Yin HM, Hsiao BS, Zhong GJ, Li ZM: Biodegradable poly(lactic acid)/hydroxyl apatite 3D porous scaffolds using high-pressure molding and salt leaching. J. Mater. Sci. 2014, 49:1648-1658.

11. Park HJ, Lee OJ, Lee MC, Moon BM, Ju HW, Lee J min, Kim JH, Kim DW, Park $\mathrm{CH}$ : Fabrication of 3D porous silk scaffolds by particulate (salt/sucrose) leaching for bone tissue reconstruction. Int. J. Biol. Macromol. 2015, 78:215223.

12. Hribar KC, Soman $P$, Warner J, Chung $P$, Chen S: Light-assisted direct-write of 3D functional biomaterials. Lab Chip 2014, 14:268-275.

- Focused review on light-assisted bioprinting technologies, in particular, digital light projection based printing and laser two photon polymerization.

13. Davey SK, Aung A, Agrawal G, Lim HL, Kar M, Varghese S: Embedded 3D Photopatterning of Hydrogels with Diverse and Complex Architectures for Tissue Engineering and Disease Models. Tissue Eng. Part C. Methods 2015, 00:1-9.

14. Liu Z, Lin Q, Sun Y, Liu T, Bao C, Li F, Zhu L: Spatiotemporally controllable and cytocompatible approach builds 3D cell culture matrix by photouncaged-thiol Michael addition reaction. Adv. Mater. 2014, 26:3912-3917.

15. Occhetta P, Sadr N, Piraino F, Redaelli A, Moretti M, Rasponi M: Fabrication of 3D cell-laden hydrogel microstructures through photo-mold patterning. Biofabrication 2013, 5:035002.

16. Wilson $\mathrm{WC}$, Boland $\mathrm{T}$ : Cell and organ printing 1: protein and cell printers. Anat. Rec. A. Discov. Mol. Cell. Evol. Biol. 2003, 272:491-496.

17. Cohen DL, Malone E, Lipson H, Bonassar LJ: Direct freeform fabrication of seeded hydrogels in arbitrary geometries. Tissue Eng. 2006, 12:1325-1335.

18. Boland T, Mironov V, Gutowska A, Roth E a, Markwald RR: Cell and organ printing 2: fusion of cell aggregates in three-dimensional gels. Anat. Rec. A. Discov. Mol. Cell. Evol. Biol. 2003, 272:497-502. 
19. Xu T, Zhao W, Zhu JM, Albanna MZ, Yoo JJ, Atala A: Complex heterogeneous tissue constructs containing multiple cell types prepared by inkjet printing technology. Biomaterials 2013, 34:130-139.

- This paper develops a versatile method for fabricating complex and heterogeneous 3D tissue constructs with multiple cell types using simultaneous ink-jet printing. This study demonstrates an advancement of ink-jet printing technology to create complex cellladen constructs since the first use of this technology in creating bio-constructs.

20. Mironov V, Visconti RP, Kasyanov V, Forgacs G, Drake CJ, Markwald RR: Organ printing: Tissue spheroids as building blocks. Biomaterials 2009, 30:21642174.

21. Mironov V, Kasyanov V, Markwald RR: Organ printing: From bioprinter to organ biofabrication line. Curr. Opin. Biotechnol. 2011, 22:667-673.

22. Faulkner-Jones A, Fyfe C, Cornelissen D-J, Gardner J, King J, Courtney A, Shu W: Bioprinting of human pluripotent stem cells and their directed differentiation into hepatocyte-like cells for the generation of mini-livers in 3D. Biofabrication 2015, 7:44102.

23. Markstedt K, Mantas A, Tournier I, Martínez Ávila H, Hägg D, Gatenholm P: 3D Bioprinting Human Chondrocytes with Nanocellulose-Alginate Bioink for Cartilage Tissue Engineering Applications. Biomacromolecules 2015, 16:1489-1496.

24. Hsieh F-Y, Lin H-H, Hsu S-H: 3D bioprinting of neural stem cell-laden thermoresponsive biodegradable polyurethane hydrogel and potential in central nervous system repair. Biomaterials 2015, 71:48-57.

25. Pati F, Jang J, Ha D-H, Won Kim S, Rhie J-W, Shim J-H, Kim D-H, Cho D-W: Printing three-dimensional tissue analogues with decellularized extracellular matrix bioink. Nat. Commun. 2014, 5:3935.

-• This paper develops a method for the bioprinting of cell-laden constructs using novel decellularized extracellular matrix ( $\mathrm{dECM}$ ) bioink. This is the first paper demonstrating the bioprinting of $\mathrm{dECM}$ materials with cells to create $\mathrm{dECM}$ structures with high cell viability and functionality.

26. Mannoor MS, Jiang Z, James T, Kong YL, Malatesta K a., Soboyejo WO, Verma N, Gracias DH, McAlpine MC: 3D printed bionic ears. Nano Lett. 2013, 13:2634-2639.

27. Soman $\mathrm{P}$, Chung $\mathrm{PH}$, Zhang a. $\mathrm{P}$, Chen S: Digital microfabrication of userdefined 3D microstructures in cell-laden hydrogels. Biotechnol. Bioeng. 2013, 110:3038-3047.

28. Michael S, Sorg H, Peck C-T, Koch L, Deiwick A, Chichkov B, Vogt PM, Reimers $\mathrm{K}$ : Tissue Engineered Skin Substitutes Created by Laser-Assisted Bioprinting Form Skin-Like Structures in the Dorsal Skin Fold Chamber in 
Mice. PLoS One 2013, 8:e57741.

29. Lu Y, Mapili G, Suhali G, Chen S, Roy K: A digital micro-mirror device-based system for the microfabrication of complex, spatially patterned tissue engineering scaffolds. [Internet]. J. Biomed. Mater. Res. A 2006, 77:396-405.

30. Zhang a P, Qu X, Soman P, Hribar KC, Lee JW, Chen S, He S: Rapid Fabrication of Complex 3D Extracellular Microenvironments by Dynamic Optical Projection Stereolithography. Adv. Mater. 2012, 24:4266-4270.

31. Connell JL, Ritschdorff ET, Whiteley M, Shear JB: 3D printing of microscopic bacterial communities. Proc. Natl. Acad. Sci. 2013, 110:18380-18385.

32. Tumbleston JR, Shirvanyants D, Ermoshkin N, Janusziewicz R, Johnson AR, Kelly D, Chen K, Pinschmidt R, Rolland JP, Ermoshkin A, et al.: Continuous liquid interface production of 3D objects. Science 2015, 347:1349-1352.

33. Hribar KC, Finlay D, Ma X, Qu X, Ondeck MG, Chung PH, Zanella F, Engler AJ, Sheikh F, Vuori K, et al.: Nonlinear 3D projection printing of concave hydrogel microstructures for long-term multicellular spheroid and embryoid body culture [Internet]. Lab Chip 2015, 15:2412-2418.

34. Cha C, Soman P, Zhu W, Nikkhah M, Camci-Unal G, Chen S, Khademhosseini A: Structural Reinforcement of Cell-Laden Hydrogels with Microfabricated Three Dimensional Scaffolds. Biomater. Sci. 2014, 2:703-709.

35. Qu X, Zhu W, Huang S, Li Y-S, Chien S, Zhang K, Chen S: Relative impact of uniaxial alignment vs. form-induced stress on differentiation of human adipose derived stem cells. Biomaterials 2013, 34:9812-8.

36. Suri S, Han L-H, Zhang W, Singh A, Chen S, Schmidt CE: Solid freeform fabrication of designer scaffolds of hyaluronic acid for nerve tissue engineering. Biomed. Microdevices 2011, 13:983-93.

37. Gou M, Qu X, Zhu W, Xiang M, Yang J, Zhang K, Wei Y, Chen S: Bio-inspired detoxification using 3D-printed hydrogel nanocomposites. Nat. Commun. 2014, 5:1-9.

- This paper presents the 3D bioprinting of hydrogel nanocomposites featuring liverinspired design for enhanced detoxification. This is a proof-of-concept that functional nanoparticles could be incorporated in 3D bioprinting for enhanced performance of biomedical devices and potentially engineered tissues.

38. Zhu W, Li J, Leong YJ, Rozen I, Qu X, Dong R, Wu Z, Gao W, Chung PH, Wang J, et al.: 3D-Printed Artificial Microfish. Adv. Mater. 2015, 27:4411-4417.

39. Liao Y, Song J, Li E, Luo Y, Shen Y, Chen D, Cheng Y, Xu Z, Sugioka K, Midorikawa K: Rapid prototyping of three-dimensional microfluidic mixers in glass by femtosecond laser direct writing. Lab Chip 2012, 12:746-9.

40. Koch L, Deiwick A, Schlie S, Michael S, Gruene M, Coger V, Zychlinski D, Schambach A, Reimers K, Vogt PM, et al.: Skin tissue generation by laser cell 
printing. Biotechnol. Bioeng. 2012, 109:1855-1863.

41. Doraiswamy A, Narayan RJ, Harris ML, Qadri SB, Modi R, Chrisey DB: Laser microfabrication of hydroxyapatite-osteoblast-like cell composites [Internet]. J. Biomed. Mater. Res. Part A 2007, 80A:635-643.

42. Zhang W, Chen S: Femtosecond laser nanofabrication of hydrogel biomaterial. MRS Bull. 2011, 36:1028-1033.

43. Zhang W, Soman P, Meggs K, Qu X, Chen S: Tuning the Poisson's Ratio of Biomaterials for Investigating Cellular Response. Adv. Funct. Mater. 2013, 23:3226-3232.

44. Auger FA, Gibot L, Lacroix D: The pivotal role of vascularization in tissue engineering. Annu. Rev. Biomed. Eng. 2013, 15:177-200.

45. Hirt MN, Hansen A, Eschenhagen T: Cardiac tissue engineering : State of the art. Circ. Res. 2014, 114:354-367.

46. Laschke MW, Vollmar B, Menger MD: Inosculation: connecting the lifesustaining pipelines. Tissue Eng. Part B. Rev. 2009, 15:455-465.

47. Miller JS, Stevens KR, Yang MT, Baker BM, Nguyen D-HT, Cohen DM, Toro E, Chen A a, Galie P a, Yu X, et al.: Rapid casting of patterned vascular networks for perfusable engineered three-dimensional tissues. Nat. Mater. 2012, 11:768-74.

48. Kolesky DB, Truby RL, Gladman a. S, Busbee T a., Homan K a., Lewis J a.: 3D bioprinting of vascularized, heterogeneous cell-laden tissue constructs. Adv. Mater. 2014, 26:3124-3130.

- This paper presents the bioprinting of vasculature network with extrusion based 3D printer and a fugitive material that is compatible to cells. This approach enables the simultaneous printing of the vasculature structure and the surrounding cells for heterogeneous cell-laden tissue constructs.

49. Zhang Y, Yu Y, Ozbolat IT: Direct Bioprinting of Vessel-Like Tubular Microfluidic Channels. J. Nanotechnol. Eng. Med. 2013, 4:020902.

50. Yu Y, Zhang Y, Ozbolat IT: A Hybrid Bioprinting Approach for Scale-Up Tissue Fabrication. J. Manuf. Sci. Eng. 2014, 136:061013.

51. Malda J, Visser J, Melchels FP, Jüngst T, Hennink WE, Dhert WJA, Groll J, Hutmacher DW: 25th Anniversary Article: Engineering Hydrogels for Biofabrication. Adv. Mater. 2013, 25:5011-5028.

52. Ozbolat IT: Bioprinting scale-up tissue and organ constructs for transplantation. Trends Biotechnol. 2015, 33:1-6. 


\section{Figure Legends}

Table 1. Comparison of the different bioprinting techniques discussed in this review $[1,9,30]$.

\begin{tabular}{|c|c|c|c|c|}
\hline & Inkjet printing & $\begin{array}{l}\text { Extrusion } \\
\text { printing }\end{array}$ & DLP printing & $\begin{array}{l}\text { Laser assisted } \\
\text { printing }\end{array}$ \\
\hline $\begin{array}{l}\text { Printing } \\
\text { process }\end{array}$ & $\begin{array}{l}\text { Serial (drop by } \\
\text { drop) }\end{array}$ & $\begin{array}{l}\text { Serial (line by } \\
\text { line) }\end{array}$ & $\begin{array}{l}\text { Parallel and } \\
\text { continuous } \\
\text { (projection } \\
\text { based) }\end{array}$ & $\begin{array}{l}\text { Serial (dot by } \\
\text { dot) }\end{array}$ \\
\hline Printing speed & Medium $(\mathrm{mm} / \mathrm{s})$ & $\begin{array}{l}\text { Slow }(10-50 \\
\mu \mathrm{m} / \mathrm{s})\end{array}$ & Fast $\left(\mathrm{mm}^{3} / \mathrm{s}\right)$ & Medium $(\mathrm{mm} / \mathrm{s})$ \\
\hline Resolution & $50 \mu \mathrm{m}$ & $5 \mu \mathrm{m}$ & $1 \mu \mathrm{m}$ & $<500 \mathrm{~nm}$ \\
\hline $\begin{array}{l}\text { Mechanical } \\
\text { integrity }\end{array}$ & $\begin{array}{l}\text { Poor due to } \\
\text { interfaces }\end{array}$ & $\begin{array}{l}\text { Poor due to } \\
\text { interfaces }\end{array}$ & $\begin{array}{l}\text { Excellent with } \\
\text { continuous } \\
\text { printing }\end{array}$ & $\begin{array}{l}\text { Poor due to } \\
\text { interfaces }\end{array}$ \\
\hline Cell viability & $>85 \%$ & $40-80 \%$ & $85-95 \%$ & $>85 \%$ \\
\hline $\begin{array}{l}\text { Material } \\
\text { choice }\end{array}$ & $\begin{array}{l}\text { Thermo/pH/pho } \\
\text { to-sensitive }\end{array}$ & $\begin{array}{l}\text { Thermo/photo- } \\
\text { sensitive }\end{array}$ & Photosensitive & Photosensitive \\
\hline
\end{tabular}


Figure 1. Inkjet and extrusion based bioprinters. (a) Schematic of inkjet bioprinter for fabricating multi-cell heterogeneous tissue constructs [19]. (b) Bright field and (c) fluorescence microscopic top views of the printed 3D multi-cellular "pie" constructs [19]. $(d-f)$ extrusion based bioprinting of heart tissue (d), cartilage tissue (e) and adipose tissue (f) with their respective dECM (scale bar, 5mm) [25]. (g) 3D printing of bionic ear with viable chondrocytes pre-seeded in alginate hydrogel, silicone, and $\mathrm{Ag}$ nanoparticles for enhanced auditory sensing [26].

Figure 2. (a) Schematic of the DLP based bioprinter - DOPsL [30]. (b) Arrays of pyramids, spirals, domes and flowers printed by DOPsL with PEGDA [30]. (c) Biomimetic vascular structure printed by DOPsL [30]. (d) Nerve conduits printed with GM-HA labeled with red fluorophore for visualization [36]. (e) Liver-inspired 3D printed hydrogel detoxifier encapsulated with PDA nanoparticles, which trap pore-forming toxins and emit red fluorescence (scale bar, $200 \mu \mathrm{m}$ ) [37].

Figure 3. (a) Schematic of the LIFT setup (left) and the printed grid structure (middle) with green fibroblasts and red keratinocytes, as well as a multilayer skin-mimicking construct with alternating red and green keratinocytes (scale bars, $500 \mu \mathrm{m}$ ) [40]. (b) TPP setup and the printed multilayer scaffold and gradient dot array (scale bars, $10 \mu \mathrm{m}$ ) [42]. (c) Negative and positive Poisson webs fabricated by TPP for real-time single cell study [43].

Figure 4. (a) Schematic of the fabrication process for vascular network combining sacrificial dissolving and cast molding [47]. (b) 3D printed multi-scale carbohydrate glass architecture (scale bars, $200 \mu \mathrm{m}$ ) [47]. (c) Endothelialization of the vascular network showing intervessel junction, vascular lumen and capillary sprouting (scale bars, $1 \mathrm{~mm}$ (left), $200 \mu \mathrm{m}$ (middle, right)) [47]. (d) Schematic of the coaxial nozzle based bioprinter of the direct printing of vessel-like cellular channels [49]. (e) Media perfusion of the printed vessels [49]. (f) Bright field microscopic image showing living cells encapsulated along the vessels [49]. 
Figure 1

(a)

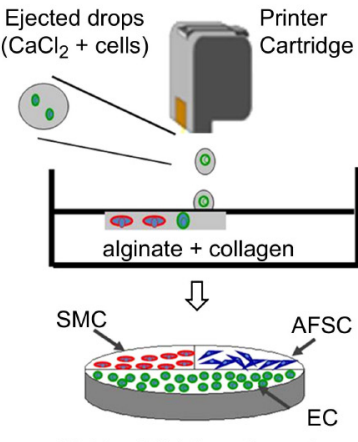

(d)

Multi-cell "pie" configuration

$$
\begin{gathered}
\text { hdECM } \\
\text { gel structure }
\end{gathered}
$$

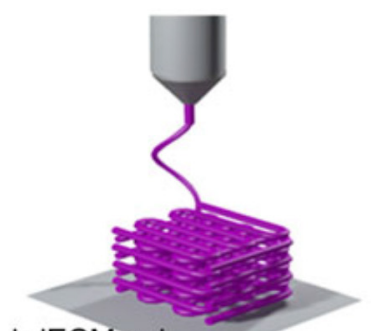

hdECM gel
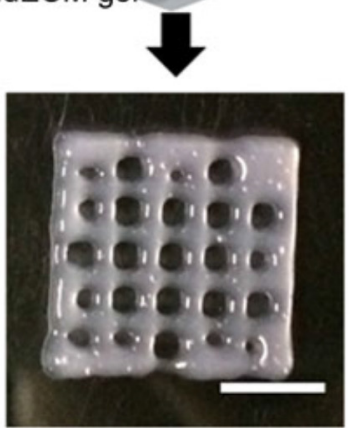

(g)

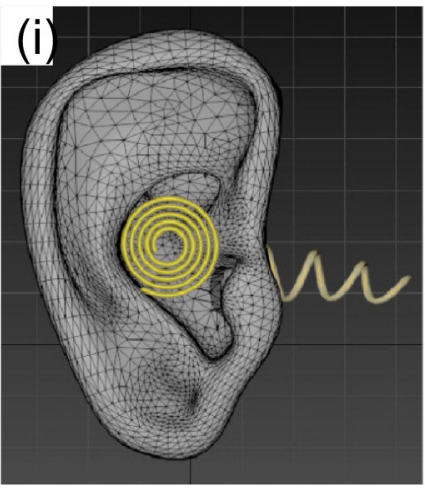

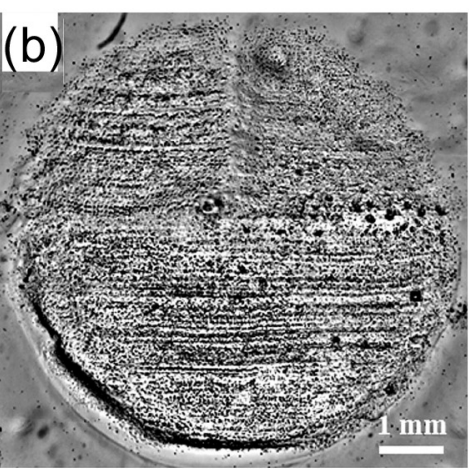

(e)

Hybrid of cdECM and PCL framework
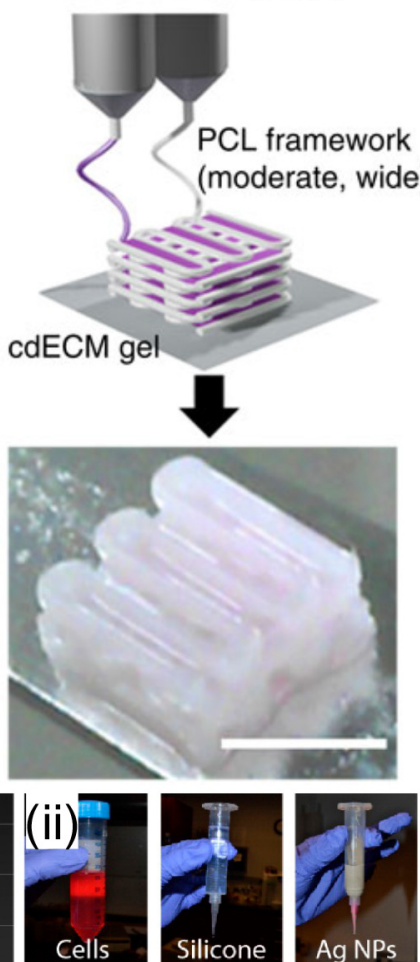

$+$

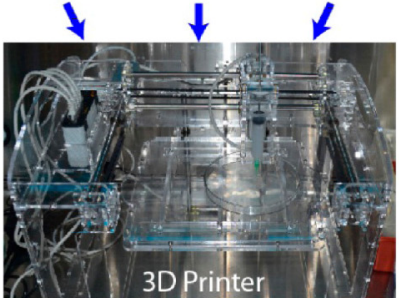

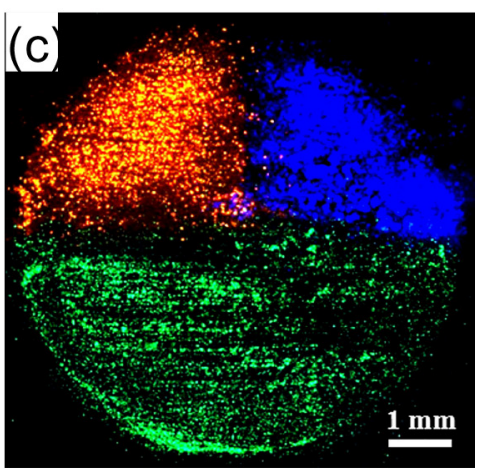

(f) Hybrid of adECM and PCL framework
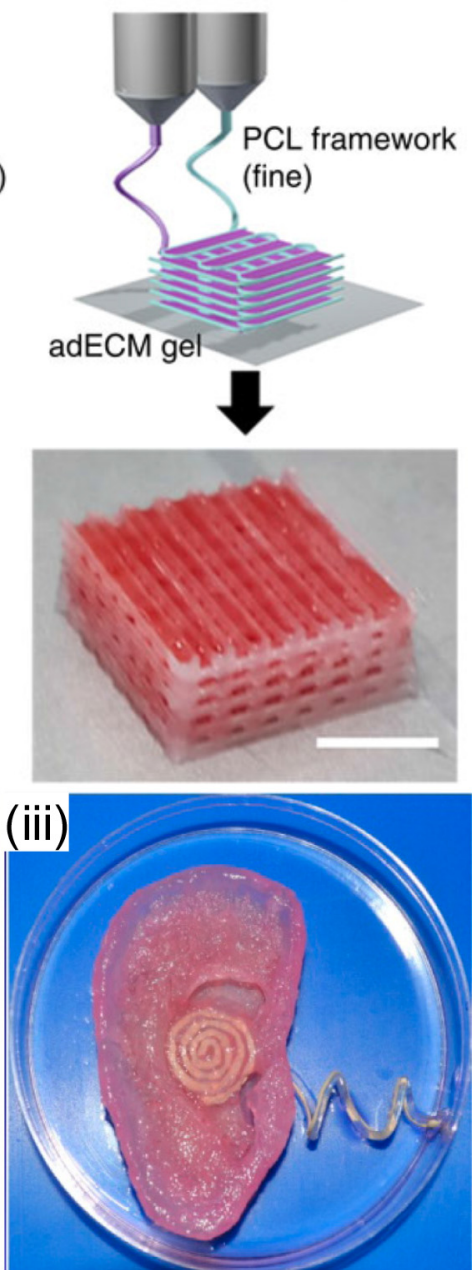
Figure 2

(a)

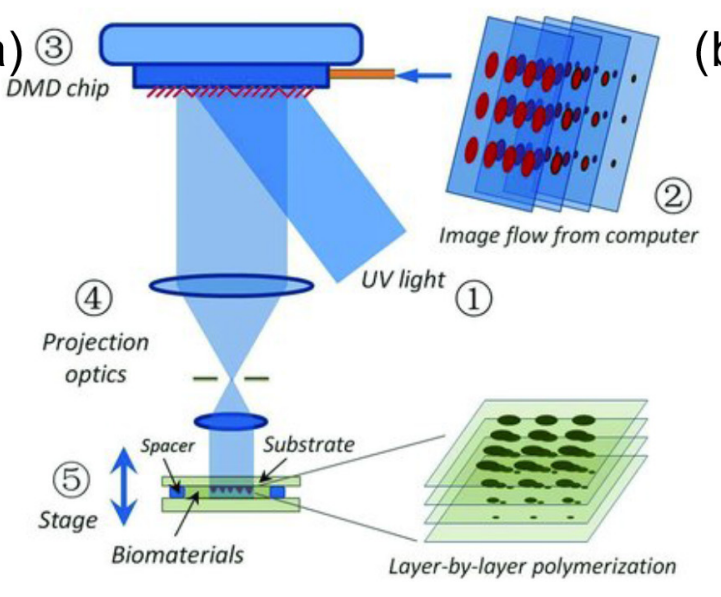

(b)
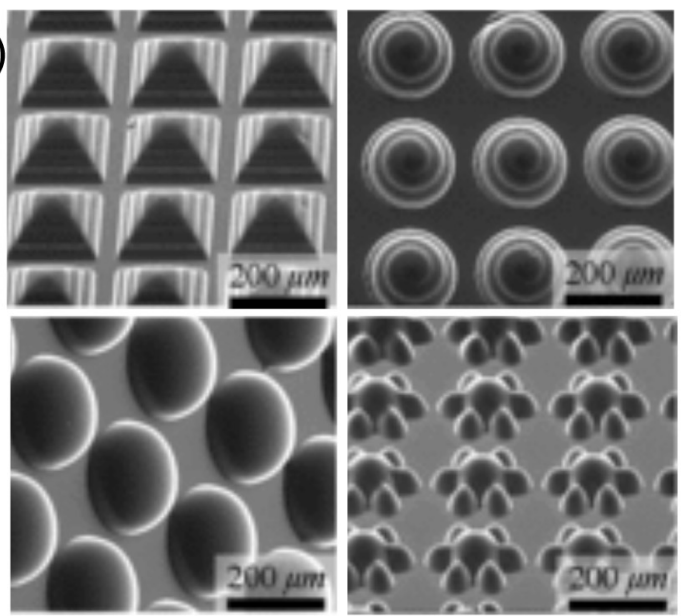

(c)

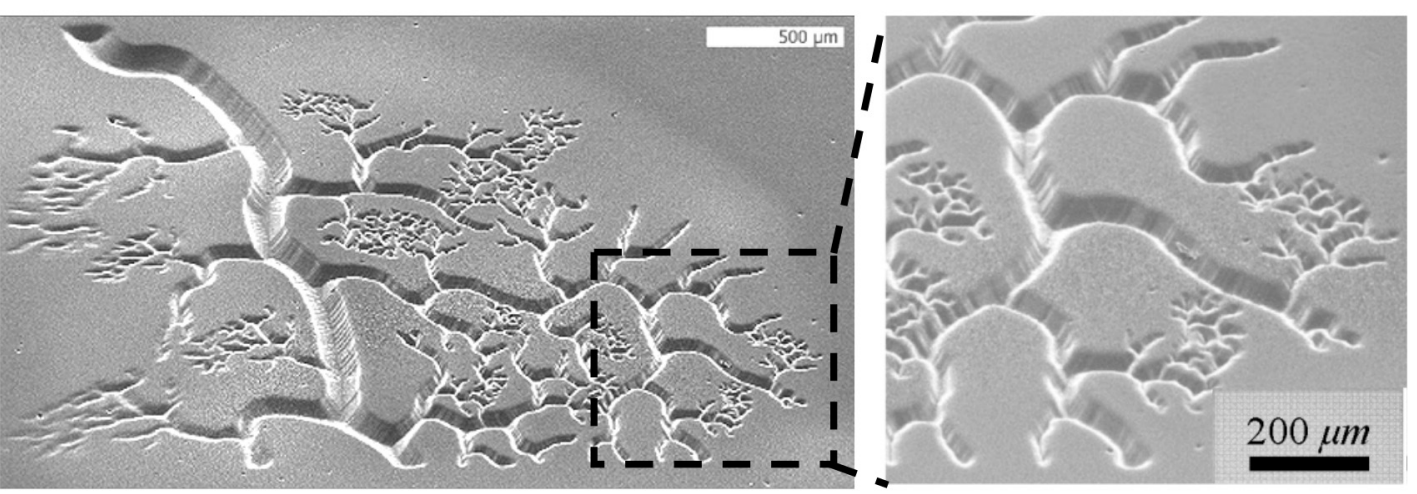

(d)
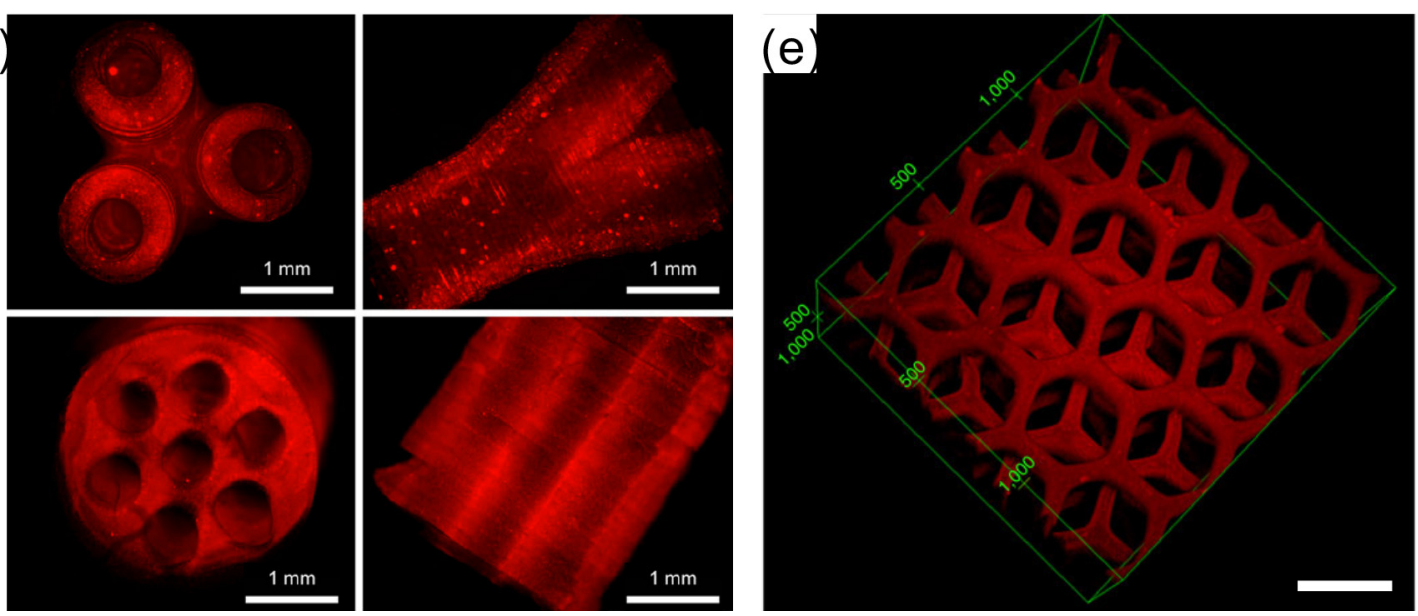
Figure 3

(a)
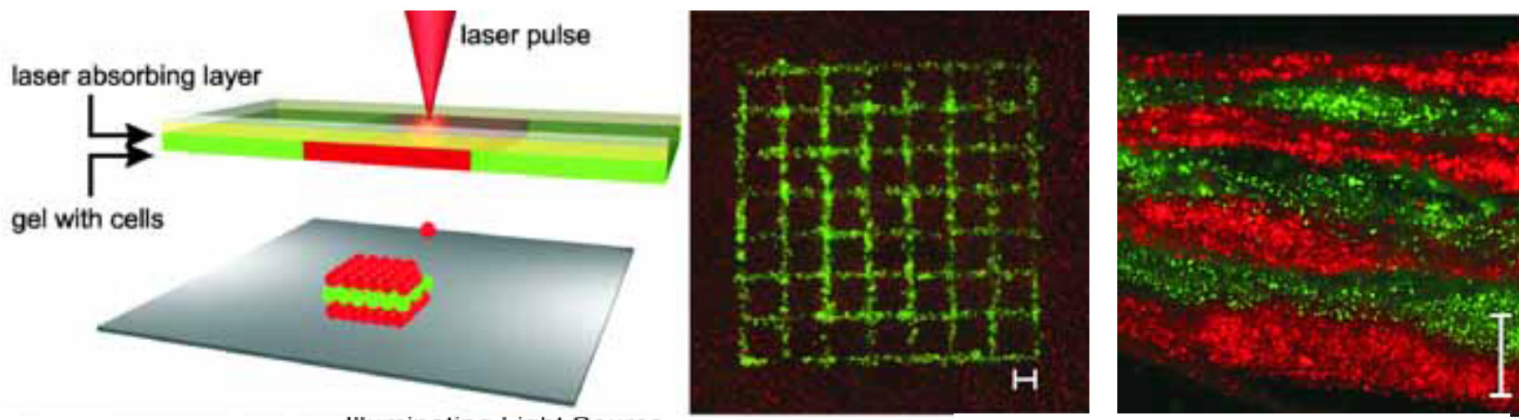

(b)

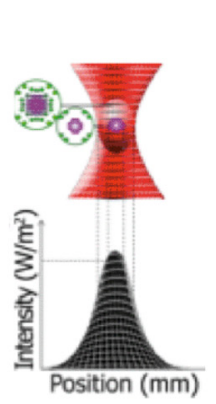

Illuminating Light Source
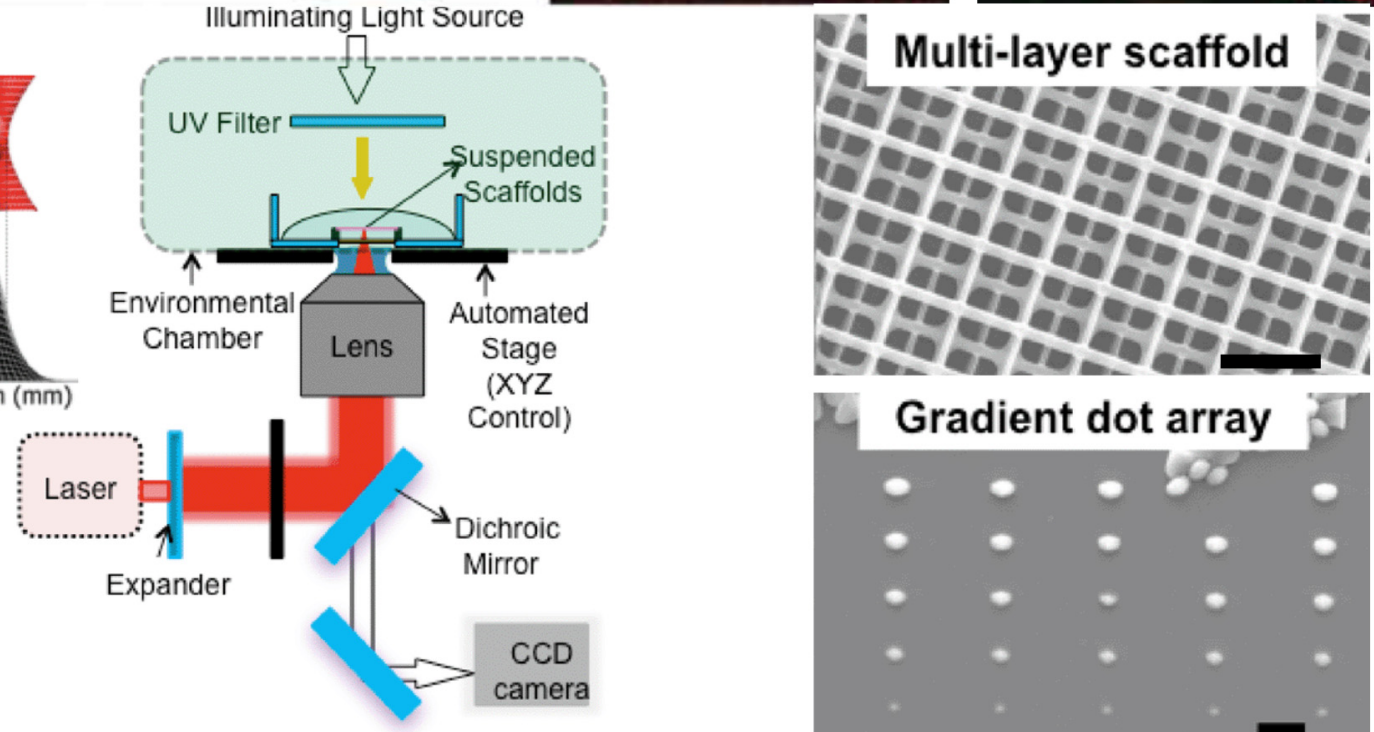

Y Gradient dot array
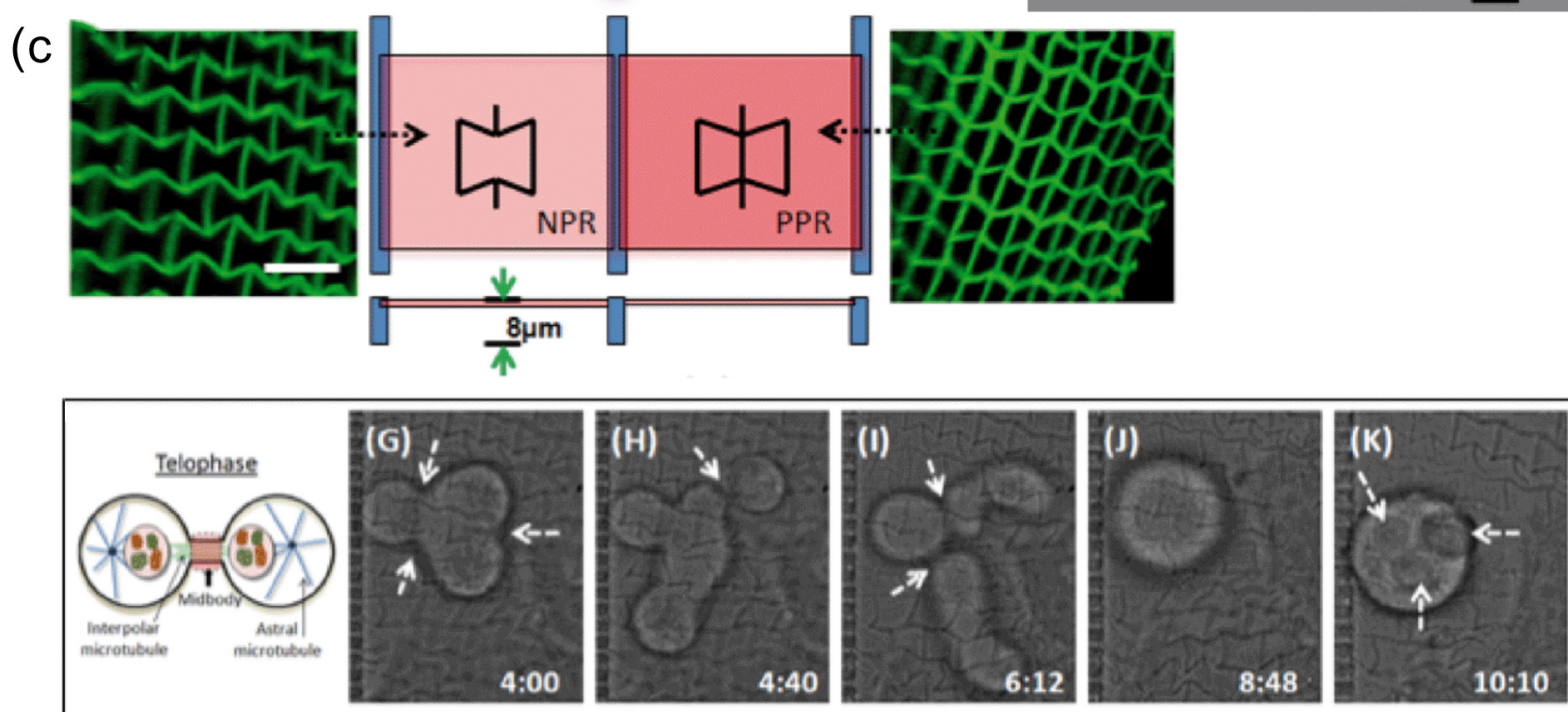
Figure 4

(a)
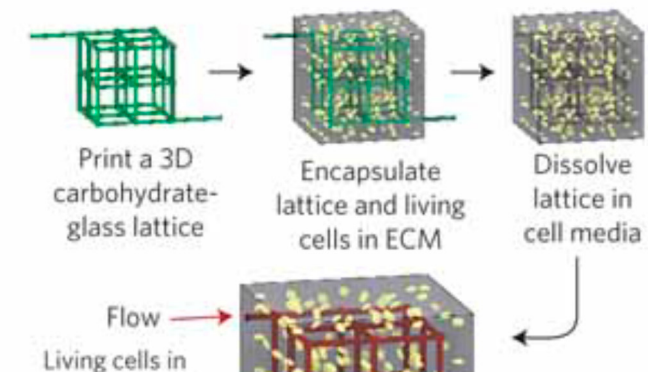

monolithic ECM

with perfusable

vascular architecture

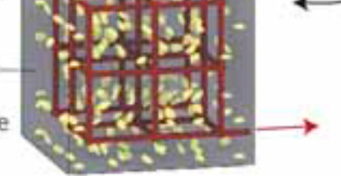

(c)

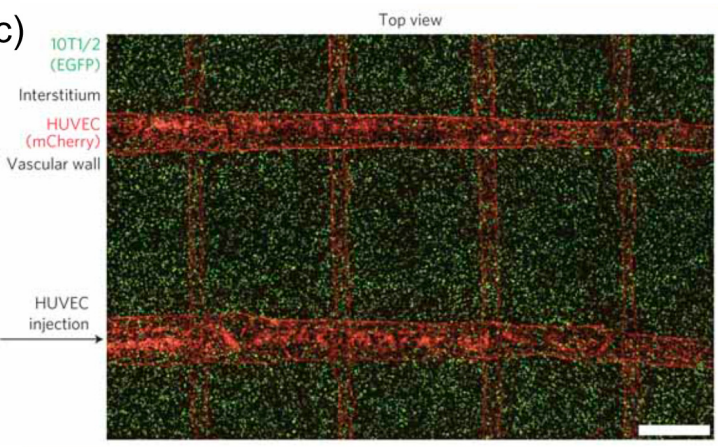

(d)

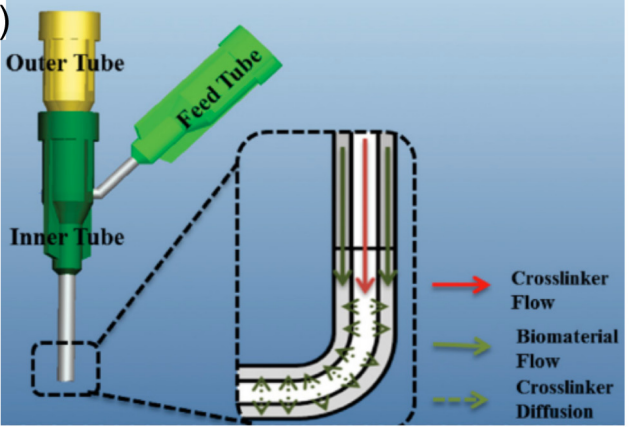

(b)
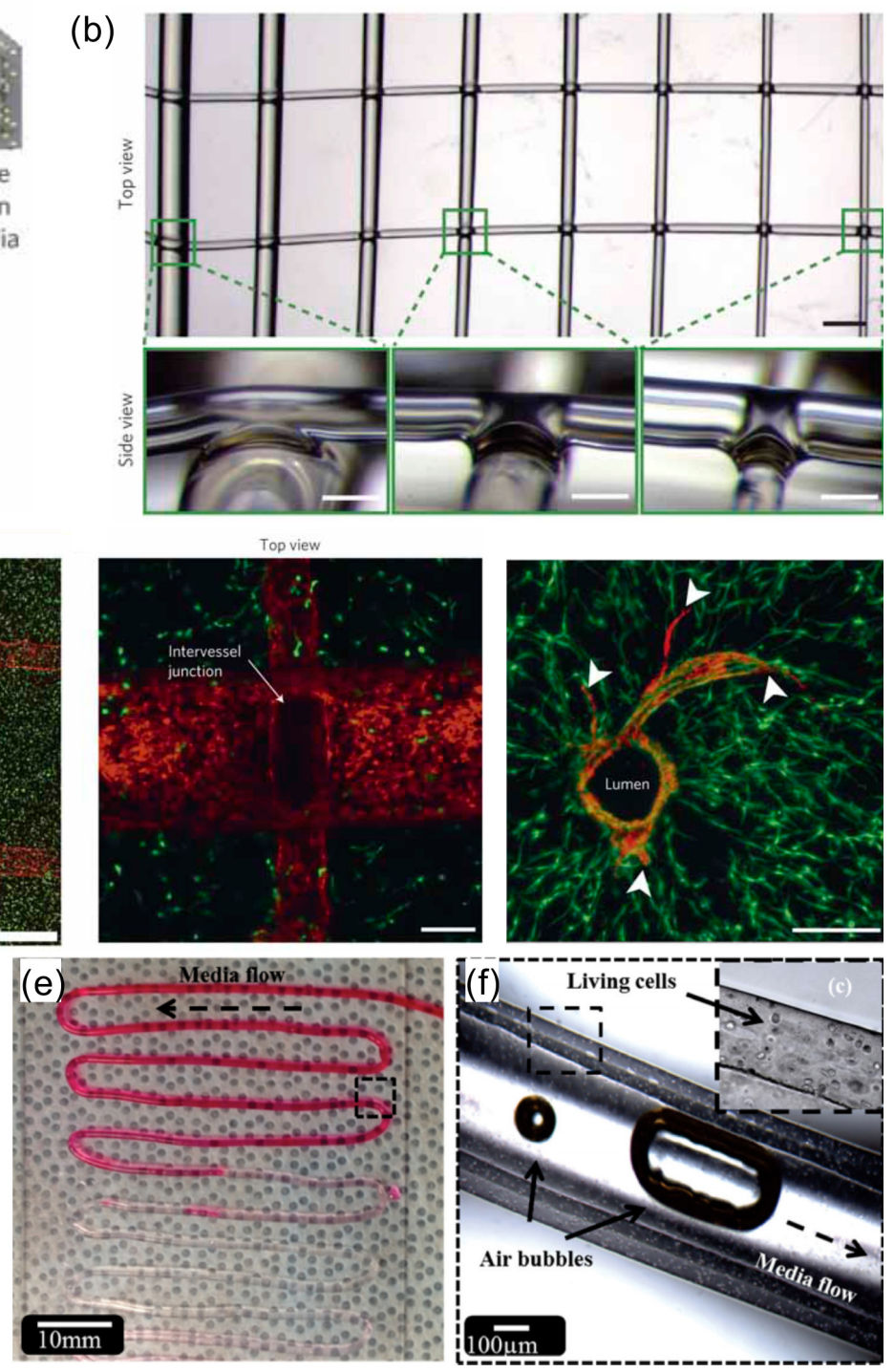
Graphical Abstract

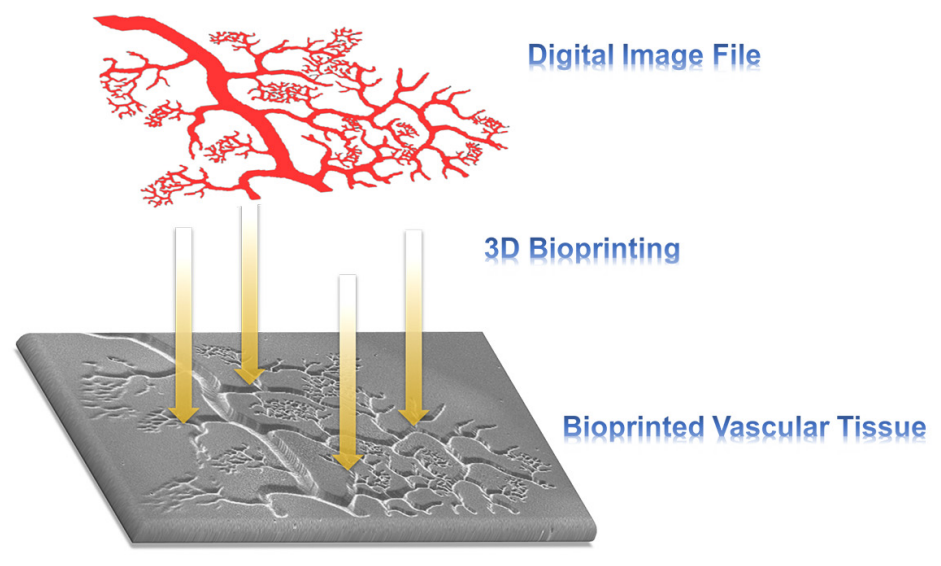

